\title{
Microarray Analysis of Long Non-Coding RNAs in Lung Tissues of Patients with COPD and HOXA-AS2 Promotes HPMECs Proliferation via Notch I
}

\author{
Ai-yuan Zhou ${ }^{1-3, *}$ \\ Yi-yang Zhao (iD) ${ }^{1-3, *}$ \\ Zi-jing Zhou ${ }^{1-3}$ \\ Jia-xi Duan ${ }^{1-3}$ \\ Yi-zhang Zhu ${ }^{4,5}$ \\ Shan Cai ${ }^{1-3}$ \\ Ping Chen ${ }^{1-3}$
}

'Department of Respiratory and Critical Care Medicine, The Second Xiangya Hospital, Central South University, Changsha, Hunan 4100II, People's Republic of China; ${ }^{2}$ Research Unit of Respiratory Disease, Central South University, Changsha, Hunan 4I00II, People's Republic of China; ${ }^{3}$ Diagnosis and Treatment Center of Respiratory Disease, Central South University, Changsha, Hunan 4100II, People's Republic of China; ${ }^{4}$ Institute of Systems Biomedicine, School of Basic Medical Sciences, Peking University Health Science Center, Beijing I00191, People's Republic of China; ${ }^{5}$ Department of Pathology, School of Basic Medical Sciences, Peking University Health Science Center, Beijing 10019I, People's Republic of China

*These authors contributed equally to this work

Correspondence: Ping Chen; Shan Cai Department of Respiratory Medicine, The Second Xiangya Hospital, Central South University, 139 Renmin Middle Road, Changsha, Hunan 4I00II, People's Republic of China

Tel +86 731 85295248

$+8673185295044$

Fax +86-73I-85295848

Email pingchen0731@csu.edu.cn; caishan@csu.edu.cn
This article was published in the following Dove Press journal:

International Journal of Chronic Obstructive Pulmonary Disease

Background and Objectives: Long non-coding RNAs (lncRNAs) play an important role in the pathogenesis of many diseases, including cancer, pulmonary fibrosis and chronic obstructive pulmonary disease (COPD). In this study, we intended to identify the differentially expressed lncRNAs and the role of HOXA cluster antisense RNA 2 (HOXA-AS2) in patients with COPD.

Methods: We analyzed lncRNA profiles of three non-COPD and seven COPD patients' lungs via microarray and then validated the expression of the top differentially expressed lncRNAs by using real-time polymerase chain reaction (PCR). To identify the mechanism of HOXA-AS2 during COPD pathogenesis and endothelial cell proliferation, we knocked down and overexpressed HOXA-AS2 with siRNA and lentivirus transfection approach in human pulmonary microvascular endothelial cells (HPMECs).

Results: Among 29,150 distinct lncRNA transcripts, 353 lncRNAs were significantly $(\geq 2$-fold change and $P<0.05$ ) upregulated and 552 were downregulated in COPD patients. The fold change of HOXA-AS2 is 9.32; real-time PCR confirmed that HOXA-AS2 was downregulated in COPD patients. In in vitro experiments, cigarette smoke extract (CSE) treatment reduced the expression of HOXA-AS2 and cell proliferation of HPMECs. Knocking down HOXA-AS2 inhibited HPMECs proliferation and the expression of Notch1 in HPMECs. Overexpressing Notch1 could partly rescue the inhibition of cell viability induced by the silence of HOXA-AS2.

Conclusion: Our results demonstrated that differentially expressed lncRNAs may act as potential molecular biomarkers for the diagnosis of COPD, and HOXA-AS2 was involved in the pathogenesis of COPD by regulating HPMECs proliferation via Notch1, which may provide a new approach for COPD treatment.

Keywords: chronic obstructive pulmonary disease, HOXA-AS2, Notch1, endothelial cell, proliferation

\section{Introduction}

Chronic obstructive pulmonary disease (COPD) is characterized by progressive and irreversible airflow limitation and associated with an abnormal inflammatory response of the lungs to noxious particles and gases, ${ }^{1}$ which causes significant morbidity and mortality worldwide and is expected to become the third leading cause of death by $2020 .^{2}$ It is known that genetic variants and environmental factors 
have a role in the pathogenesis of this disease. Smoking and/or some other chronic exposure to irritants plays an important role among the environmental factors. ${ }^{3-5}$ However, only $20 \%$ of smokers develop COPD ${ }^{6}$ indicating that genetic and epigenetic factors contribute to the susceptibility for this disease.

Protein-coding genes only represent a small part of the human genome $(20,687$ genes, $<2 \%){ }^{7}$ while a large portion is transcribed into non-coding RNAs (ncRNAs), including microRNAs (miRNAs) and long non-coding RNAs (lncRNAs), both of which were originally thought to be non-functional. With the advancement of high-resolution microarray analysis methods, genome-wide sequencing technology or RNA-sequencing, the role of non-coding RNA has generated considerable interest. It was demonstrated that lncRNAs were involved in various cellular functions, including cell proliferation, cell growth, cell differentiation and apoptosis. ${ }^{8-10}$ The abnormal expression of lncRNAs was revealed in many diseases, including cancer, pulmonary fibrosis, and COPD. ${ }^{1-15}$ Recently, increasing evidence has shown that HOXA cluster antisense RNA 2 (HOXA-AS2), a lncRNA located between and antisense to the human HOXA3 and HOXA4 genes, is associated with cell survival, proliferation and invasion. ${ }^{16-18}$ Moreover, it is reported that expression of HOXA-AS2 is related to endothelium inflammation. ${ }^{19}$ In our previous studies, we found the dysfunction of endothelial cells was involved in the pathogenesis of COPD. ${ }^{20,21}$ However, whether HOXAAS2 has a role in the progress of COPD by regulating the function of endothelial cells remains unclear.

In this study, we detected the expression of IncRNAs in the lung tissues of three non-COPD and seven COPD patients by Agilent microarray 3.0 system. We found that HOXA-AS2 was significantly downregulated in COPD patients, and HOXA-AS2 can regulate human pulmonary microvascular endothelial cells (HPMECs) proliferation. Meanwhile, we further investigated the mechanism by which HOXA-AS2 was involved in the pathogenesis of COPD and regulating HPMECs proliferation.

\section{Patients and Methods Lung Tissue Samples}

This study was approved by the Institutional Ethics Committee of the Second Xiangya Hospital of Central South University $(2,016,032)$ and was performed in accordance with the ethical standards established in the 1964 Declaration of Helsinki and its later amendments or comparable ethical standards. All the patients provided informed consent. COPD patients $(n=7)$ and non-COPD patients $(n=3)$ were diagnosed as spontaneous pneumothorax and required pulmonary segmentectomy or lobectomy. The diagnosis of COPD was based on the Global Initiative for Chronic Obstructive Lung Disease (GOLD) 2013. ${ }^{22}$ All the patients were in a clinically stable state without pulmonary infection in the last 4 weeks. Patients with other chronic pulmonary diseases, such as obstructive atelectasis and asthma, lung cancer, and other systemic diseases, including diabetes, cardiovascular diseases, renal diseases, hepatic diseases or mental diseases, were excluded. Lung tissue samples were immediately frozen in liquid nitrogen for further experiments, or fixed in $4 \%$ formaldehyde and cut into 3.5 -mm-thick sections to stain with hematoxylin and eosin (HE).

\section{Arraystar IncRNA Array}

Lung tissue RNA was isolated using Trizol reagent (Life Technologies Corporation, CA, USA), and RNA quality was determined. RNA labeling reaction was performed using a Quick Amp Labeling Kit, One-Color ( $\mathrm{p} / \mathrm{n}$ 5190-0442, Agilent Technologies, CA, USA) and purified by RNeasy Mini Kit (p/n 74,104, Qiagen, Hilden, Germany). Then, labeled/amplified cRNA was hybridized using Agilent Gene Expression Hybridization Kit (p/n 5188-5242, Agilent), and then the microarray was washed. We used Agilent Microarray Scanner (p/n G2565BA, Agilent) and Agilent Feature Extraction to scan and extract data. (Aksomics Inc., Shanghai, China)

\section{Cell Culture}

HPMECs were purchased from ScienCells Research Laboratory (CA, USA) and were cultured in endothelial cell medium (ECM, ScienCells Research Laboratory, CA, USA) supplemented with 5\% fetal bovine serum (FBS), $1 \%$ endothelial cell growth factor and penicillin (100 IU/ $\mathrm{mL}) /$ streptomycin $(50 \mu \mathrm{g} / \mathrm{mL})$ at $37^{\circ} \mathrm{C}$ in a humidified incubator, containing 5\% CO2. Only cells at passages 3 to 4 were used in experiments.

\section{CSE Preparation}

CSE was prepared as described previously. ${ }^{20}$ Briefly, one commercial cigarette (Furong, Changde Cigarette Company, Hunan, China) without filter was burned, and the smoke passed through $25 \mathrm{~mL}$ of basic ECM without FBS and endothelial cell growth factor by a vacuum pump. Then, the smoke extract was filtered through a $0.22-\mu \mathrm{m}$ pore-size filter. The PH of the solution was adjusted to 7.2-7.4. The product 
was regarded as $100 \%$ CSE solution and subsequently diluted with complete ECM to obtain final concentrations of $0.5 \%$, $1 \%, 2.5 \%$, and $5 \%$ in in vitro study.

\section{Cell Transfection}

Using Lipofectamine 2000 (Invitrogen, CA, USA) according to the manufacturer's instructions, HOXA-AS2 siRNA and respective negative control (NC) siRNA (RiboBio Corporation, Guangzhou, China), Notch1 overexpression plasmid vectors and empty vectors (GeneChem, Shanghai, China) were transfected into endothelial cells. For HOXAAS2 overexpression lentivectors (GeneChem, Shanghai, China) infection, firstly we co-cultured the lentivectors with different cell density in different medium with or without Polybrene or ENi.S.(Enhanced Infection Solution) to find the proper multiplicity of infection (MOI) for lentivectors. Then, the selected condition, including cell seeding density and the most effective conditioned medium, was used in the subsequent infection experiments. The representative images of cell transduction efficiency by lentivectors were shown in Supplementary Material: Figure S1.

\section{Cell Proliferation Assay}

The cells were seeded into 96 wells, and treated with the HOXA-AS2 siRNA, or CSE at different concentrations of 0 , $0.5 \%, 1 \%, 2.5 \%$, and $5 \%$, or $10 \mathrm{mM}$ of Notch 1 inhibitor DAPT (GSI-IX, gamma-Secretase Inhibitor IX, ApexBio Technology, TX, USA) for 24h. Then, cell proliferation was carried out by Cell Counting Kit-8 (CCK-8, Dojindo Laboratories, Kumamoto, Japan) according to the manufacturer's protocol. In brief, after treatment, the culture medium was aspirated and changed into fresh medium. Then, $10 \mu \mathrm{L}$ of CCK-8 reagent was added into each well and incubated at $37^{\circ} \mathrm{C}$ for $4 \mathrm{~h}$. The absorbance at $450 \mathrm{~nm}$ was measured by a microplate reader, which determined the survival rate of endothelial cells.

\section{Real-Time Polymerase Chain Reaction (Real-Time PCR)}

Total RNA was extracted from lung tissues, and cells were cultured using Trizol reagent (Life Technologies Corporation, CA, USA). RNA was reverse transcribed using RevertAid ${ }^{\mathrm{TM}}$ First Strand cDNA Synthesis Kit (Thermo Fisher Scientific, USA). Then, real-time PCR was performed with UltraSYBR Mixture (ComWin Biotech Corporation, Beijing, China) following the manufacturer's protocol in PIKO REAL 96 PCR System (Thermo Fisher Scientific, USA). The sequence of primers is shown in the Supplementary Material: Table S1. We used GAPDH as the internal loading control and calculated the relative expression of IncRNA or mRNA by $2^{-\Delta \Delta C T}$ method. Each PCR analysis was done in triplicate.

\section{Western Blot}

Total protein was extracted from cells by RIPA lysis (Beyotime Institute of Biotechnology, Jiangsu, China) containing $1 \%$ protease/phosphatase inhibitor cocktail (ComWin Biotech Corporation, Beijing, China) and then measured by Pierce BCA Protein Assay Kit (Thermo Fisher Scientific, USA) to determine the protein concentration. Protein lysates were loaded and separated in 4-20\% sodium dodecyl sulfate polyacrylamide gel electrophoresis (SDSPAGE) gel (Sigma, USA) and transferred to polyvinylidene difluoride (PVDF) membranes after electrophoresis (EMD Millipore, Billerica, MA, USA). After blocking with 5\% nonfat dry milk for $1 \mathrm{~h}$ at room temperature, the membranes were washed and incubated with Notch1 and glyceraldehyde 3-phosphate dehydrogenase (GADPH) primary antibody (20,687-1-AP and 10,494-1-AP, Proteintech Group Incorporation, IL, USA) in dilutions of 1:1000 and 1:3000 at $4{ }^{\circ} \mathrm{C}$ overnight. The next day, the membranes were washed and incubated with horseradish peroxidase-labeled secondary antibody (SA00001-2, Proteintech Group Incorporation, IL, USA) at a 1:5000 dilution. Then, the antibody-labeled bands were detected by an enhanced chemiluminescence detection system (ECL, BestBio Pharmacia Biotech). The results were quantified with Quantity One Analysis Software (Bio-Rad Laboratories, Hercules, CA, 210 USA).

\section{Statistical Analysis}

Statistical analysis of data was conducted by SPSS 22.0 (SPSS Incorporation, IL, USA) and GraphPad Prism 6.0 (GraphPad Software, CA, USA). One-way analysis of variance (ANOVA) was used to analyze the difference between two groups and the least significant difference (LSD) post hoc test was performed to compare the differences between multiple groups. There was statistical significance when $\mathrm{p}$-values were less than 0.05 .

\section{Results \\ Overview of the IncRNA and mRNA Profiles in Lung Tissues of COPD}

Three non-COPD and seven COPD patients were selected for this study. The basic characteristics are shown in Table 1. 
Table I Clinical Characteristics of the Subjects Recruited for the Microarray

\begin{tabular}{|l|l|l|l|l|l|l|}
\hline Number & Group & Age & Sex & $\begin{array}{l}\text { Pack- } \\
\text { Years }\end{array}$ & FEVI\% & $\begin{array}{l}\text { FEVII } \\
\text { FVC }\end{array}$ \\
\hline 1 & Non-COPD & 65 & Male & 20 & 81.0 & 99.0 \\
2 & Non-COPD & 53 & Female & 0 & 118.0 & 82.0 \\
3 & Non-COPD & 67 & Male & 0 & 77.7 & 73.4 \\
4 & COPD & 64 & Male & 10 & 51.4 & 49.7 \\
5 & COPD & 61 & Female & 30 & 79.0 & 47.0 \\
6 & COPD & 51 & Male & 20 & 80.0 & 61.0 \\
7 & COPD & 68 & Female & 10 & 69.2 & 55.9 \\
8 & COPD & 64 & Male & 40 & 62.0 & 54.0 \\
9 & COPD & 66 & Male & 20 & 56.0 & 50.0 \\
10 & COPD & 69 & Male & 40 & 70.4 & 65.8 \\
\hline
\end{tabular}

Abbreviations: COPD, chronic obstructive pulmonary disease; FEVI, forced expiratory volume in I second; FVC, forced vital capacity.

The hematoxylin-eosin (HE) staining of COPD and nonCOPD lung tissues were performed (Supplementary Material: Figure S2). LncRNA array was used to measure the differentially expressed IncRNAs in COPD and nonCOPD lung tissues. Twenty-five thousand six hundred and six distinct lncRNAs transcripts were detected in the lung tissues of all subjects. In COPD patients, 353 lncRNAs were significantly ( $\geq 2$-fold change and $\mathrm{P}<0.05$ ) up-regulated, and 552 were downregulated when compared to patients without COPD (Figure 1A). RP11-160E2.19, LUCAT1, LINC01108, LY86-AS1, and GO63948 were the top five upregulated lncRNAs with the fold change ranging from 4.67 to 12.23. RP5-1158E12.1, G052118, G057815, Uc.128-, and G089388 were the top five downregulated IncRNAs with the fold change ranging from 12.92 to 15.35 (Figure 1B). Eighteen thousand eight hundred and ninety-four distinct mRNA transcripts were detected in the lung tissues of all subjects. In COPD patients, 270 mRNAs were significantly $(\geq 2$-fold change and $\mathrm{P}<0.05)$ upregulated and 293 were downregulated when compared to patients without COPD (Figure 1C and D).

\section{Real-Time PCR Validation}

We selected the top five upregulated lncRNAs and six downregulated lncRNAs based on the fold change for confirmation of expression in another two sets of lung tissue samples using real-time PCR, which includes RP11-160E2.19, LUCAT1, LINC01108, LY86-AS1, GO63948, RP51158E12.1, G052118, G057815, Uc.128-, G089388, and HOXA-AS2. The basic characteristics of the patients are seen in Supplementary Material: Table S2. The results indicated $63.64 \%$ consistency between the microarray data and real-time PCR results. RP11-160E2.19, LUCAT1, GO63948, RP5-1158E12.1, G052118, uc.128-, and HOXAAS2 were confirmed to change significantly between COPD patients and non-COPD patients (Table 2).

\section{Kyoto Encyclopedia of Genes and Genomes (KEGG) Pathway Analysis and Gene Ontology Enrichment Analysis}

Pathway analysis demonstrated that there were several enrichment-related pathways. It included 34 upregulated pathways (the top ten up-regulated pathways are shown in Figure 2A), such as the cytokine-cytokine receptor interaction, chemokine signaling pathway, Toll-like receptor signaling pathway, and NF-kappa B signaling pathway. Ten downregulated pathways (Figure 2B) included protein digestion and absorption, steroid hormone biosynthesis, retinol metabolism, and drug metabolism-cytochrome P450.

Gene ontology (GO) analysis was performed to determine the gene product enrichment (http://www.geneontol ogy.org). Three domains in ontology were analyzed: biological process (Figure $2 \mathrm{C}$ and $\mathrm{D}$ ), cellular component (Figure 2E and $\mathrm{F}$ ), and molecular function (Figure 2G and $\mathrm{H}$ ). For the biological process, there were 526 upregulated and 401 downregulated GO items between the COPD and non-COPD groups. Immune response and tissue development had the highest enrichment score value of the significant enrichment terms.

\section{CSE Treatment Inhibited HPMECs Proliferation and Reduced the Expression of HOXA-AS2}

HPMECs were exposed to CSE at different concentrations of $0.0 \%, 0.5 \%, 1.0 \%, 2.5 \%$, and $5.0 \%$ for $24 \mathrm{~h}$. CCK- 8 assay was then performed to examine cell viability. The results demonstrated that at the concentration of $2.5 \%$ CSE, both the cell viability and the expression level of HOXA-AS2 were decreased significantly (Figure $3 \mathrm{~A}$ and $\mathrm{B}$ ). Then, we explored the optimum timepoint for the experiment. The expression of HOXA-AS2 decreased significantly at $12 \mathrm{~h}$, $24 \mathrm{~h}$, and $48 \mathrm{~h}$ (Figure 3C). CSE at a concentration of $2.5 \%$ was chosen for the following experiment.

\section{Effects of HOXA-AS2 on the HPMECs Proliferation}

To examine the effect of HOXA-AS2 on cell proliferation, silencing and overexpression approaches were used. We 
A

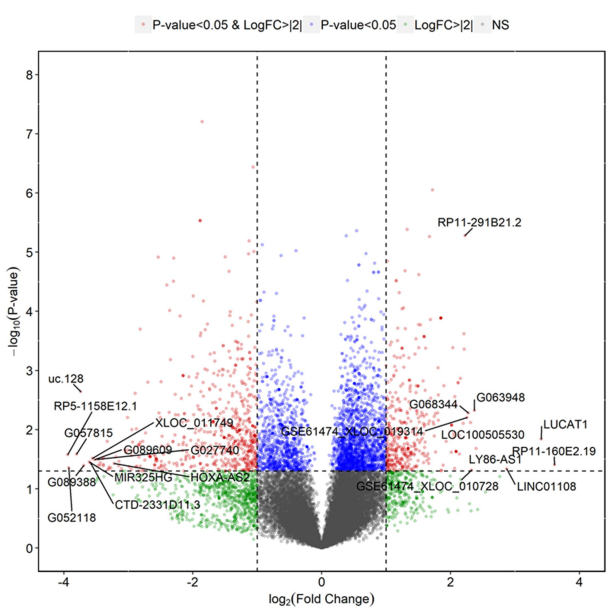

C

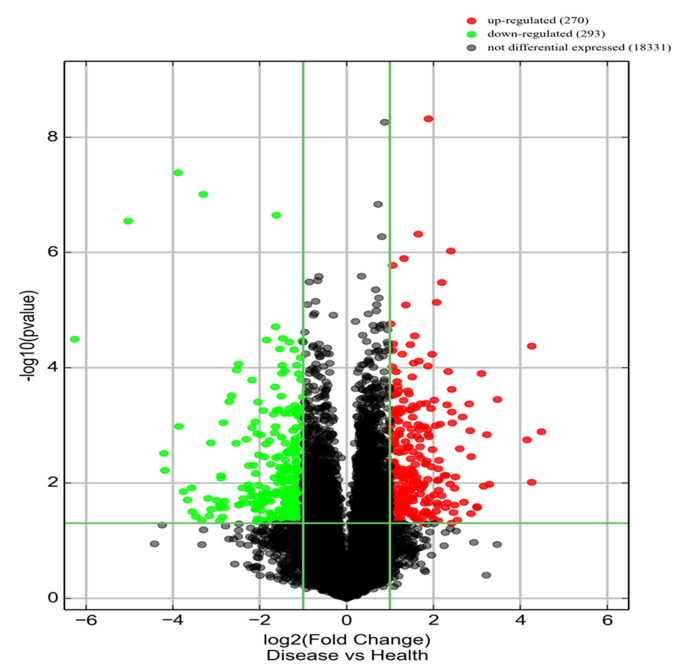

B

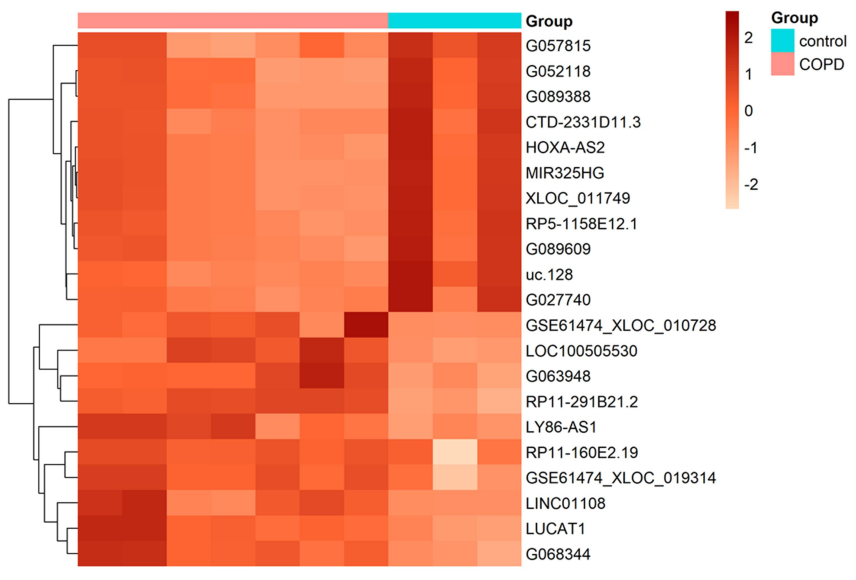

D

1-3 Controls without COPD 4-10 Patients with COPD

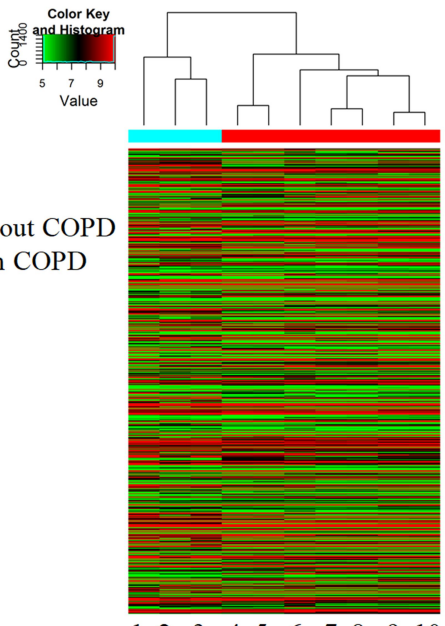

$\begin{array}{llllllllll}1 & 2 & 3 & 4 & 5 & 6 & 7 & 8 & 9 & 10\end{array}$

Figure I The differential expression of IncRNAs and mRNA in COPD and non-COPD lung tissues. (A) The volcano plot of differential expressed IncRNAs in COPD compared to non-COPD lung tissues. The expressions of IncRNAs were presented in red ( $\geq 2$-fold change and $P<0.05$ ), green ( $\geq 2$-fold change but $P \geq 0.05$ ), blue ( $\leq 2$-fold change and $P<0.05$ ), and gray (NS). (B) Hierarchical clustering showed the top differential expression of IncRNAs in COPD (red bar) and non-COPD (blue bar) lung tissues. The expression was displayed on a scale from light (low) to deep (high). (C) The volcano plot of differentially expressed mRNAs in COPD compared to non-COPD lung tissues. The expression of upregulated mRNAs was presented in red ( $\geq 2$-fold change and $P<0.05$ ), downregulated in green ( $\geq 2$-fold change and $P<0.05)$ and gray (NS). (D) The hierarchical clustering of all differential expressed mRNAs in COPD (red bar) compared to non-COPD (blue bar) lung tissues. The expression was displayed on a scale from green (low) to red (high).

Abbreviations: COPD, chronic obstructive pulmonary disease; IncRNAs, long non-coding RNAs; NS, no significance.

knocked down HOXA-AS2 in HPMECs by transfecting with three kinds of siRNA and found that all the siRNAs caused a significant decrease in HOXA-AS2 expression (Figure 4A). Because siRNA2 transfection had more profound effects on suppressing HOXA-AS2 expression than the other two siRNAs, we chose siRNA2 for the following experiment. The cell viability was significantly inhibited after being transfected with siRNA for $24 \mathrm{~h}$ (Figure 4B). To further clarify the role of HOXA-AS2 in regulating cell viability, we overexpressed HOXA-AS2 in HPMECs via transfecting with lentivirus and found that overexpression of HOXA-AS2 could alleviate cell viability inhibition induced by CSE treatment (Figure 4C).

\section{HOXA-AS2 Regulated HPMECs Proliferation via Notch I}

To investigate the mechanisms by which HOXA-AS2 regulates cell proliferation, we used real-time PCR to detect the expression of some important genes related to endothelial proliferation, including Notch1, Fli1, Wnt3a, and vascular endothelial growth factor (VEGF). We found that only Notch 1 was significantly decreased after knocking 
Table 2 The Verification of Microarray Analysis Selected from Different Expressed Long Non-Coding RNAs in the List

\begin{tabular}{|l|l|l|l|l|}
\hline LncRNA* & Expression & Fold Change & P value (Microarray) & P value (Real-Time PCR Verification) \\
\hline RPII-I60E2.19 & UP & 12.23 & 0.04 & 0.014 \\
LUCATI & UP & 10.60 & 0.01 & 0.036 \\
LINC0II08 & UP & 7.31 & 0.04 & 0.549 \\
LY86-ASI & UP & 5.27 & 0.02 & 0.067 \\
G063948 & Up & 4.67 & 0.02 & 0.025 \\
RP5-II58EI2.I & Down & 15.35 & 0.03 & 0.007 \\
G052II8 & Down & 15.21 & 0.04 & 0.009 \\
G0578I5 & Down & 13.97 & 0.03 & 0.262 \\
uc. I28- & Down & 13.35 & 0.002 & 0.008 \\
G089388 & Down & 12.92 & 0.04 & 0.455 \\
HOXA-AS2 & Down & 9.32 & 0.04 & 0.014 \\
\hline
\end{tabular}

Note: *LncRNAs, long non-coding RNAs.

down HOXA-AS2 (Figure 5A). The other three genes did not present significant differences between the control and HOXA-AS2 siRNA group (Supplementary Material: Figure S3). Western blot also confirmed that knocking down HOXA-AS2 downregulated the expression of Notch1 following the same trend (Figure 5B). In addition, we found that CSE treatment could decrease the expression of Notch1, and it could be partly reversed by overexpressing HOXA-AS2 (Figure 5C). We further confirmed the function of Notch1 in regulating cell proliferation. Notch1 inhibitor and Notch1 overexpression plasmid were used to investigate the functional role of Notch1 in HPMECs. Our results demonstrated that Notch1 inhibitor decreased the expression of Notch1, while Notch1 overexpression could increase the expression of Notch1 (Figure 5D). Importantly, the inhibition of Notch1 decreased cell viability significantly, whereas overexpression of Notch1 could promote the proliferation of HPMECs, suggesting that Notch1 was involved in cell cycle regulation (Figure 5E). To further address the role of Notch1 in HOXA-AS2 regulating cell proliferation, we overexpressed Notch1 in HPMECs after transfecting with HOXA-AS2 siRNA for $24 \mathrm{~h}$. This showed that overexpression of Notch1 could partly rescue the cell viability inhibition induced by the silence of HOXA-AS2 (Figure 5F). Notch1 inhibitor could reduce the cell viability promoted by overexpressing HOXA-AS2 (Figure 5G).

\section{Discussion}

The present study showed that lncRNA HOXA-AS2 was significantly downregulated in COPD lung tissues and CSEtreated HPMECs. The expression of HOXA-AS2 showed a dose- and time-dependent decrease in HPMECs when exposed to CSE. We also found that HOXA-AS2 promoted proliferation of HPMECs. Moreover, Notch1 was found to be a downstream molecule of HOXA-AS2, and overexpressing Notch1 protected HPMECs against the damage of cell survival induced by CSE and HOXA-AS2 silencing. Taken together, our study demonstrated that HOXA-AS2 was involved in preservation of endothelial cell viability during the pathogenesis of COPD through regulating Notch1. Our findings strongly suggest the importance of lncRNAs in COPD pathogenesis and likely provide a new target in COPD treatment.

Over the past decades, IncRNAs have been proven to be essential regulators of transcription and translation by their ability to interact with DNAs, RNAs, and proteins. ${ }^{23-25}$ Moreover, the dysregulation of lncRNAs is associated with pathological processes of different diseases, such as cardiovascular diseases, metabolic diseases, and cancer. ${ }^{26-28}$ However, the role that lncRNAs plays in COPD pathogenesis remains to be determined. To address this problem, we used microarray analysis to study the differential expression of lncRNAs in COPD and non-COPD lung tissues. Three hundred fifty-three lncRNAs were upregulated, and 552 IncRNAs were downregulated in COPD lung tissues compared to non-COPD tissues. These results suggested that there were significant changes in IncRNA profile in COPD pathological processes. Also, we found that hundreds of mRNAs were significantly differentially expressed in COPD lungs. The GO and pathway analyses showed that IncRNAs was involved in various signaling pathways and metabolic process, such as the NF-kappa B signaling pathway, Wnt/ $\beta$-catenin signaling pathway, inflammatory response, and immune system. Early studies have shown that NF-kappa B plays an important role in COPD 
A

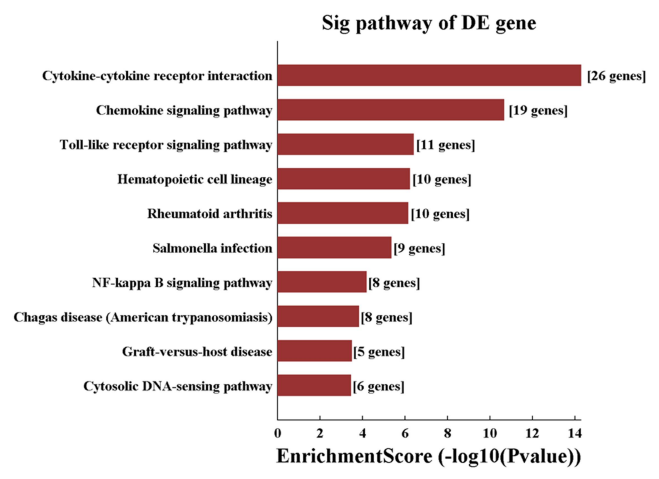

C

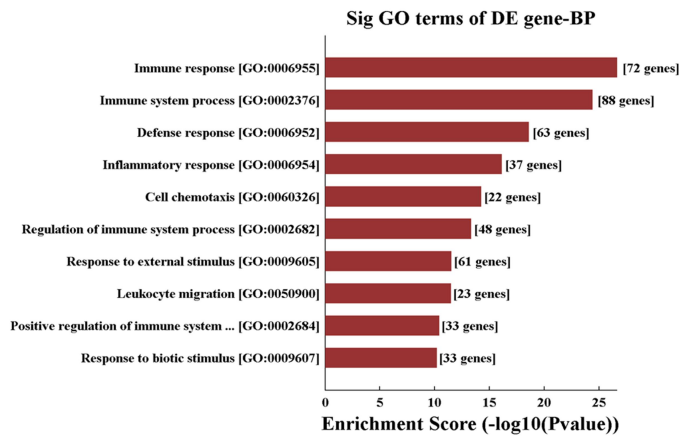

E

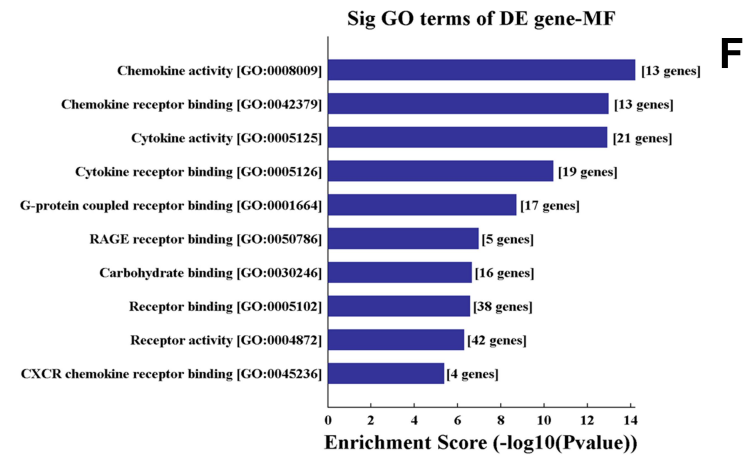

$\mathbf{F}$
B
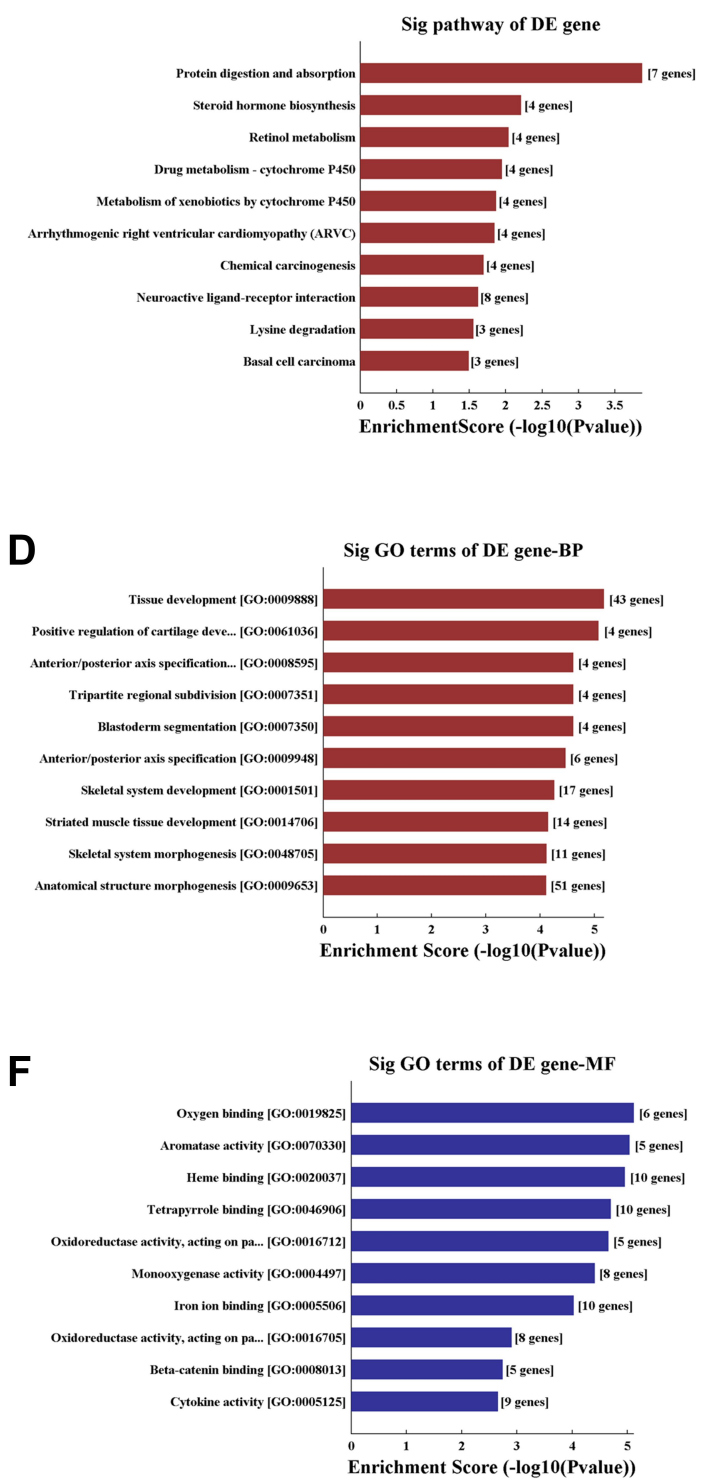

H

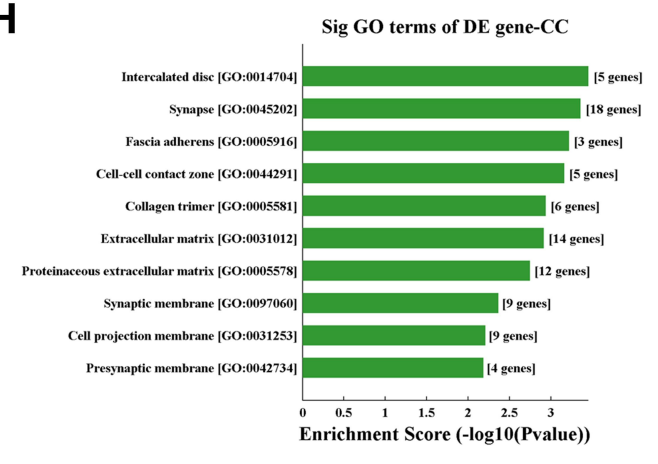

Figure 2 KEGG pathway analysis and GO enrichment analysis of differentially expressed mRNAs. (A and B) The significantly upregulated (A) and downregulated (B) pathways in COPD vs non-COPD patients. The P-value (EASE-score, Fisher's P-value, or hypergeometric P-value) denotes the significance of the pathway correlated to the conditions. The lower the $\mathrm{P}$-value, more significant is the pathway (the recommended $P$-value cutoff is $\mathbf{0 . 0 5}$ ). (C and $\mathbf{D}) \mathrm{GO}$ analysis about the top ten counts of enrichment score in the biological process. Up-regulated (C) and down-regulated (D) in the biological process. (E and $\mathbf{F}$ ) GO analysis of the top ten counts of enrichment score in the biological process. Up-regulated (E) and down-regulated (F) gene molecular function. ( $\mathbf{G}$ and $\mathbf{H}) \mathrm{GO}$ analysis of the top ten counts of enrichment score of up-regulated $(\mathbf{G})$ and down-regulated $(\mathbf{H})$ cellular component. Abbreviations: COPD, chronic obstructive pulmonary disease; KEGG pathway analysis, Kyoto Encyclopedia of Genes and Genomes pathway analysis; GO enrichment analysis, gene ontology enrichment analysis. 
A

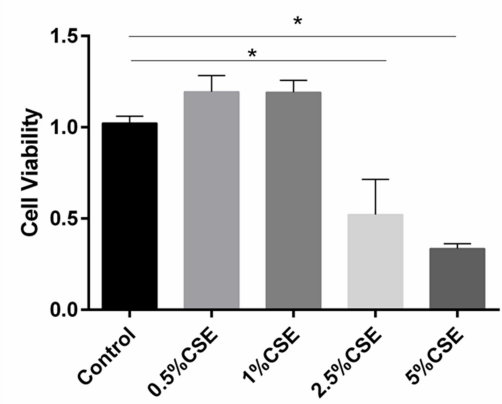

B

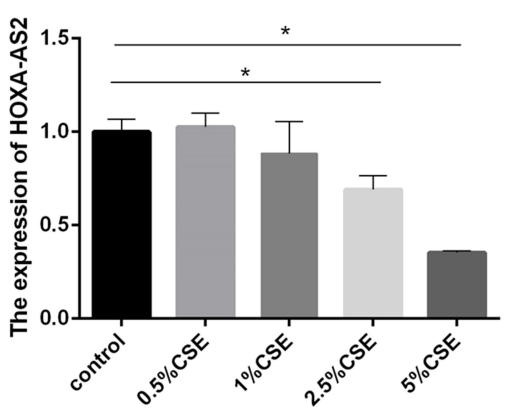

C

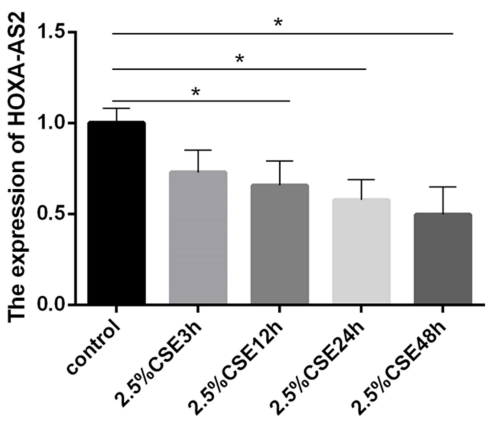

Figure 3 CSE treatment inhibited the proliferation of HPMECs and reduced HOXA-AS2 expression. (A) HPMECs were treated with different concentrations of CSE, including $0,0.5 \%, 1.0 \%, 2.5 \%$, and $5.0 \%$ for $24 \mathrm{~h}$, and the proliferation of HPMECs was measured by CCK- 8 assay. (B) HPMECs was exposed to CSE of different concentrations ( $0,0.5 \%$, $1.0 \%, 2.5 \%$, and $5.0 \%$ ) for $24 \mathrm{~h}$, and the relative expression levels of HOXA-AS2 was measured by real-time-PCR. At the concentrations of $2.5 \%$ and $5 \%$ CSE, the cell viability decreased significantly. (C) HPMECs were treated with 2.5\% CSE for different amounts of time, and the relative expression levels of HOXA-AS2 were measured by real-time PCR. The expression of HOXA-AS2 decreased significantly as the treatment time extended. Data are presented as mean \pm SEM. $* P<0.05$. We used GAPDH as the internal loading control and calculated the relative expression of IncRNA by $2^{-\triangle \Delta C T}$ method. All experiments were performed independently at least 3 times.

Abbreviations: CSE, cigarette smoke extract; real-time PCR, real-time polymerase chain reaction; HPMECs, human pulmonary microvascular endothelial cells; CCK-8, Cell Counting Kit-8.

A

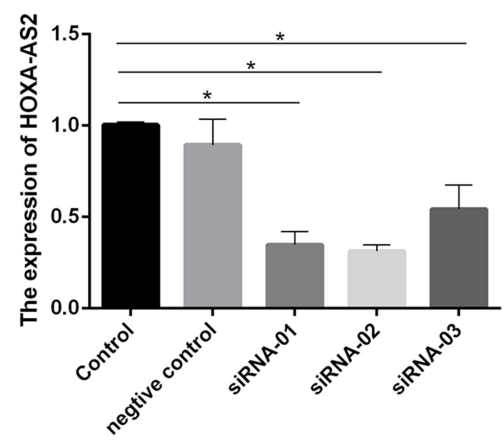

B

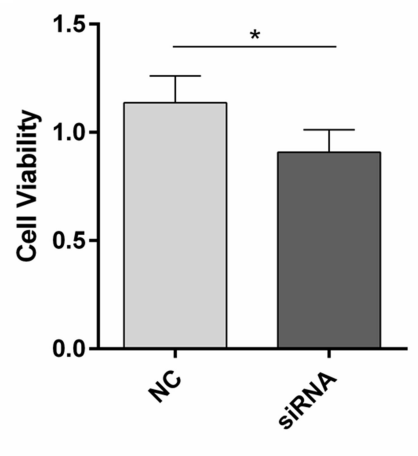

C

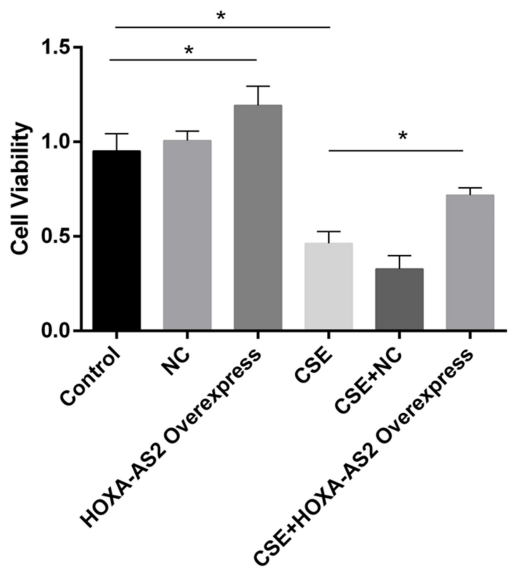

Figure 4 HOXA-AS2 promoted the proliferation of HPMECs. (A) Three kinds of siRNAs were transfected into HPMECs, and the relative expression levels of HOXA-AS2 were measured by real-time PCR. All the siRNAs could cause significant decrease in HOXA-AS2 expression. (B) HPMECs were transfected with negative control (NC) and HOXA-AS2 siRNA for $24 \mathrm{~h}$, and cell viability was measured by CCK-8 assay. This showed that the silence of HOXA-AS2 decreased the proliferation of HPMECs. (C) After transfection with HOXA-AS2 overexpression lentiviral vectors and empty lentiviral vectors, HPMECs were incubated with $2.5 \%$ CSE for 24 h. Then, the cell viability was measured by CCK-8 assay. The results suggested that HOXA-AS2 promoted the viability of HPMECs and partly rescued the damage of cell proliferation induced by CSE. All data are presented as mean \pm SEM. $* P<0.05$. We used GAPDH as the internal loading control and calculated the relative expression of IncRNA by $2^{-\triangle \Delta C T}$ method. All experiments were performed independently at least 3 times.

Abbreviations: HPMECs, human pulmonary microvascular endothelial cells; real-time PCR, real-time polymerase chain reaction; CCK-8, Cell Counting Kit-8. CSE, cigarette smoke extract.

pathogenesis through regulating several inflammatory mediators that are essential to the development of disease. ${ }^{29}$ The Wnt/ $\beta$-catenin signaling pathway has also been widely accepted to be related to the destruction of parenchymal tissue and emphysema repair. ${ }^{30}$ In addition, recent studies have uncovered that immune response has a potential role in COPD pathogenesis. ${ }^{31}$ Therefore, our study supported that IncRNAs could be involved in COPD progression through various signaling pathways.
Furthermore, the real-time PCR validation showed that lncRNA HOXA-AS2 was significantly downregulated in COPD lung tissue. Recent studies have demonstrated that HOXA-AS2 promotes cell proliferation, migration, and invasion in various human cancers, such as breast cancer, bladder cancer, and osteosarcoma. ${ }^{16,17,32}$ Zhu et al ${ }^{19}$ reported that inhibiting HOXA-AS2 could activate the nuclear factor (NF)- $\mathrm{\kappa B}$ signaling pathway and induce subsequent inflammatory response in endothelial cells. 
A

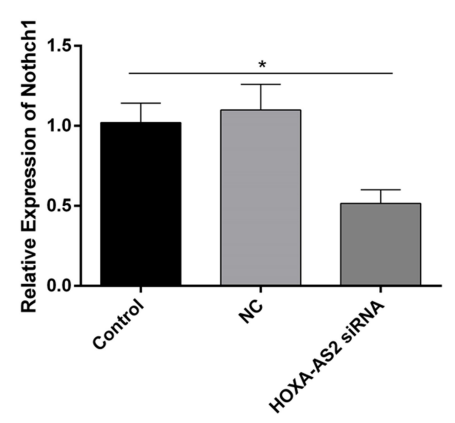

B

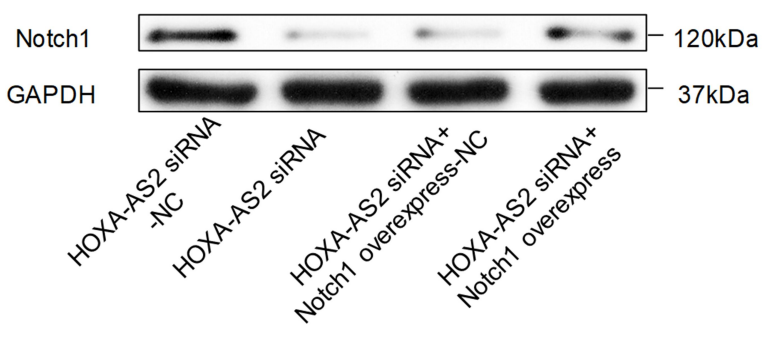

D

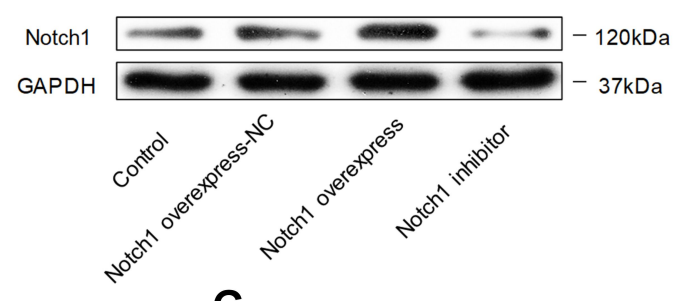

G
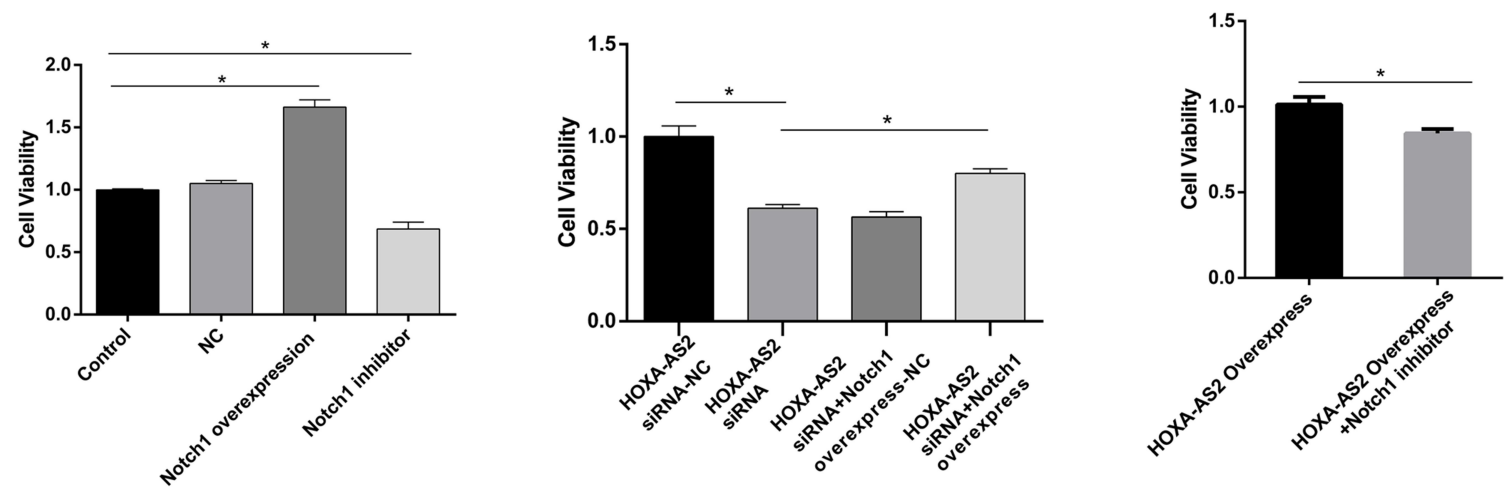

Figure 5 HOXA-AS2 promote HPMECs proliferation through regulating Notchl. (A) HOXA-AS2 siRNAs were transfected into HPMECs for 24 h, and the relative expression levels of Notch I were measured by real-time PCR. Notchl mRNA decreased in HOXA-AS2 knocking down HPMECs. (B-D) Western blot was used to analyze the expression of Notchl protein after transfection with HOXA-AS2 siRNA, overexpressing HOXA-AS2 lentivirus, NotchI overexpression plasmid or NotchI inhibitor gamma-Secretase Inhibitor IX (GSI-IX, DAPT 10 mM for $24 \mathrm{~h}$ ) in HPMECs. (E-G) Cell Counting Kit-8 (CCK-8) assay was used to measure HPMECs viability after treatment. All data are presented as mean \pm SEM. $* P<0.05$. All experiments were performed independently at least 3 times.

Abbreviations: HPMECs, human pulmonary microvascular endothelial cells; real-time-PCR, real-time polymerase chain reaction; CCK-8, Cell Counting Kit-8.

Endothelium dysfunction is one of the most essential factors contributing to COPD pathogenesis, which has been confirmed by our previous studies. ${ }^{20,21,33}$ Given the importance of endothelial cells in COPD, we detected the role of HOXA-AS2 in pulmonary endothelial cells. The results showed that the expression of HOXA-AS2 was decreased in HPMECs when exposed to CSE, in dose- and timedependent reduction. These results revealed that HOXAAS2 expression within HPMEC level is regulated by CSE stimuli. Mechanistically, HOXA-AS2 silencing suppressed endothelial cell survival, while overexpressing HOXAAS2 attenuates the viability damage of HPMECs induced by CSE. These results all suggested that HOXA-AS2 could promote the viability of HPMECs, but the mechanisms which were involved in the process needed to be further investigated.

To explore the mechanism of HOXA-AS2 on regulating the proliferation of HPMECs, we searched the online database AnnoLnc (http://annolnc.cbi.pku.edu.cn/) to determine the HOXA-AS2 target protein. Unfortunately, there was no predictional co-expressed protein that was matched in differentially expressed mRNA in microarray analyses. Then, we tried to identify some other important genes related to endothelial proliferation, such as Notch1, Fli1, Wnt3a, and VEGF. The results showed that only Notch 1 was significantly decreased after knocking down HOXAAS2 in HPMECs. Notch1 has been widely accepted to have significant effects on cell fate determination, including cell 
proliferation, differentiation, and cell apoptosis. ${ }^{34}$ When Notch1 is activated by ligands located on the cell surface, Notch1 undergoes intra-membrane proteolysis, then it releases an intracellular region which induces the downstream signaling cascade. ${ }^{35}$ Our previous study reported that Notch1 was downregulated in HPMECs when exposed to CSE both in vivo and in vitro. ${ }^{20}$ Concordantly with these early studies, we found that Notch 1 overexpression promoted endothelial cell proliferation, whereas NOTCH1 inhibitor decreased cell viability. Interestingly, the expression of Notch1 decreased after knocking down HOXA-AS2 and increased in endothelial cells with HOXA-AS2 overexpression. These data suggested that Notch1 mediates HOXA-AS2-dependent proliferation of HPMECs.

Our study has potential limitations. First, in this study, we verified the top five upregulated lncRNAs, five downregulated IncRNAs, and HOXA-AS2. However, there were hundreds of IncRNAs differentially expressed in COPD lung tissues, and these lncRNAs may also have significant effects in COPD pathogenesis. Second, the present study demonstrated that HOXA-AS2 attenuated the damage of cell viability induced by CSE and promoted proliferation of HPMECs via Notch1. But the mechanism by how HOXA-AS2 regulates Notch1 remains unknown. Third, there was no evidence about the effects of HOXAAS2 on endothelial cells in an animal model in our study, but the analysis of human lung tissues and thorough study of HOXA-AS2 in endothelial cells can provide strong background information on animal study.

\section{Conclusions}

In summary, our study revealed the differential expression level of lncRNAs between COPD and non-COPD lung tissues through microarray analysis. GO and pathway analyses could help us explore and predict the function of certain lncRNAs. The results also suggested that HOXAAS2 promotes cell proliferation and attenuates the damage of cell viability induced by CSE exposure through upregulation of Notch1. Our findings not only highlight an important role of HOXA-AS2 in the proliferation of HPMECs, but also provide novel insights into searching effective therapeutic targets.

\section{Abbreviations}

COPD, chronic obstructive pulmonary disease; CSE, cigarette smoke extract; ECM, endothelial cell media; FEV1, forced expiratory volume in 1 second; FVC, forced vital capacity; GOLD, Global Initiative for Chronic Obstructive
Lung Disease; HOXA-AS2, HOXA cluster antisense RNA 2; HPMEC, human pulmonary microvascular endothelial cell; LncRNA, long non-coding RNA; PCR, polymerase chain reaction.

\section{Data Sharing Statement}

The authors confirm that the data supporting the findings of this study are available within the article. The datasets used and/or analyzed during the current study are available from the corresponding author upon reasonable request. The authors will not share any individual de-identified participant data or other relevant study documents.

\section{Ethics Approval and Consent to Participate}

This research was approved by the Institutional Ethics Committee of the Second Xiangya Hospital of Central South University $(2,016,032)$, and all subjects provided written informed consent to participate in the study.

\section{Acknowledgments}

The authors thank all the patients who participated in the study. Thank Dr. Zong Dandan (Department of Respiratory and Critical Care Medicine, the Second Xiangya Hospital, Central South University, Changsha, Hunan 410011, China) for her help in providing NOTCH1 overexpression plasmid vectors and thanks Dr. Guo Peipei (Pulmonary, Critical Care and Sleep Medicine, Icahn School of Medicine at Mount Sinai, New York 11766, USA) for helping us check the revised manuscript carefully to make sure the language was accurate and clear enough.

\section{Author Contributions}

PC, AZ, and YYZ contributed to the study conceptualization; $\mathrm{ZZ}, \mathrm{YYZ}$, and $\mathrm{AZ}$ provided patient lung tissues and clinical information. AZ, ZY, and YZZ contributed to data analysis. AY and YZ performed PCR validation. YYZ and YZ performed the cell experiments. JD and $Z Z$ analyzed the CCK-8 and Western blot data. PC, AZ, and YYZ contributed to funding acquisition. $\mathrm{AZ}$ and $\mathrm{YYZ}$ contributed to the drafting of the manuscript. PC and SC contributed to critically revising the manuscript for important intellectual content. All authors contributed to data analysis, drafting or revising the article, have agreed on the journal to which the article will be submitted, gave final 
approval of the version to be published, and agree to be accountable for all aspects of the work.

\section{Funding}

This work was supported by the National Natural Science Foundation (NSFC) of China under Grant 81770046 to Prof Ping Chen; Fundamental Research Funds for the Central South University under Grant 2017zzts228 to Dr. Ai-yuan Zhou; Hunan Provincial Innovation Foundation For Postgraduate under Grant CX20190157 to Dr. Yi-yang Zhao.

\section{Disclosure}

All authors declare no conflicts of interest for this work.

\section{References}

1. Pauwels RA, Buist AS, Calverley PM, Jenkins CR, Hurd SS, Committee GS. Global strategy for the diagnosis, management, and prevention of chronic obstructive pulmonary disease. NHLBI/WHO Global Initiative for Chronic Obstructive Lung Disease (GOLD) workshop summary. Am J Respir Crit Care Med. 2001;163 (5):1256-1276. doi:10.1164/ajrccm.163.5.2101039

2. World Health Organization. World health statistics 2008. Available from: http://www.who.int/whosis/whostat/EN_WHS08_Full.pdf. Accessed May 20, 2008.

3. Ezzati M. Indoor air pollution and health in developing countries. Lancet. 2005;366(9480):104-106. doi:10.1016/S0140-6736(05)66845-6

4. Li LJ, Gao LB, Lv ML, et al. Association between SNPs in pre-miRNA and risk of chronic obstructive pulmonary disease. Clin Biochem. 2011;44(10-11):813-816. doi:10.1016/j.clinbiochem.2011.04.021

5. Trupin L, Earnest G, San Pedro M, et al. The occupational burden of chronic obstructive pulmonary disease. Eur Respir J. 2003;22 (3):462-469. doi:10.1183/09031936.03.00094203

6. Pauwels RA, Rabe KF. Burden and clinical features of chronic obstructive pulmonary disease (COPD). Lancet. 2004;364 (9434):613-620. doi:10.1016/S0140-6736(04)16855-4

7. Consortium EP. An integrated encyclopedia of DNA elements in the human genome. Nature. 2012;489(7414):57-74.

8. Zhang L, Zhou C, Qin Q, Liu Z, Li P. LncRNA LEF1-AS1 regulates the migration and proliferation of vascular smooth muscle cells by targeting miR-544a/PTEN axis. $J$ Cell Biochem. 2019;120 (9):14670-14678.

9. Huang T, Wang J, Zhou Y, Zhao Y, Hang D, Cao Y. LncRNA CASC2 is upregulated in osteoarthritis and participates in the regulation of IL-17 expression and chondrocyte proliferation and apoptosis. Biosci Rep. 2019;39(5). doi:10.1042/BSR20182454

10. Delas MJ, Jackson BT, Kovacevic T, et al. IncRNA Spehd regulates hematopoietic stem and progenitor cells and is required for multilineage differentiation. Cell Rep. 2019;27(3):719-729 e716. doi:10.1016/j.celrep.2019.03.080

11. Zhao Q, Zhao S, Li J, et al. TCF7L2 activated HOXA-AS2 decreased the glucocorticoid sensitivity in acute lymphoblastic leukemia through regulating HOXA3/EGFR/Ras/Raf/MEK/ERK pathway. Biomed Pharmacother. 2019;109:1640-1649. doi:10.1016/j.biopha. 2018.10.046

12. Wang X, Cheng Z, Dai L, et al. Knockdown of lncRNA H19 represses the progress of pulmonary fibrosis through the TGF-beta/ Smad3 pathway by regulating miR-140. Mol Cell Biol. 2019;39(12). doi:10.1128/MCB.00143-19.
13. Schattin A, Arner R, Gennaro F, de Bruin ED. Adaptations of prefrontal brain activity, executive functions, and gait in healthy elderly following exergame and balance training: a randomized-controlled study. Front Aging Neurosci. 2016;8:278. doi:10.3389/fnagi.2016.00278

14. Gu C, Li Y, Liu J, et al. LncRNAmediated SIRT1/FoxO3a and SIRT1/p53 signaling pathways regulate type II alveolar epithelial cell senescence in patients with chronic obstructive pulmonary disease. Mol Med Rep. 2017;15(5):3129-3134. doi:10.3892/ mmr.2017.6367

15. Thai $\mathrm{P}$, Statt $\mathrm{S}$, Chen $\mathrm{CH}$, Liang E, Campbell C, Wu R. Characterization of a novel long noncoding RNA, SCAL1, induced by cigarette smoke and elevated in lung cancer cell lines. Am J Respir Cell Mol Biol. 2013;49(2):204-211. doi:10.1165/rcmb.2013-0159RC

16. Wang $\mathrm{F}$, Wu D, Chen J, et al. Long non-coding RNA HOXA-AS2 promotes the migration, invasion and stemness of bladder cancer via regulating miR-125b/Smad2 axis. Exp Cell Res. 2019;375(1):1-10. doi:10.1016/j.yexcr.2018.11.005

17. Wang Y, Zhang R, Cheng G, Xu R, Han X. Long non-coding RNA HOXA-AS2 promotes migration and invasion by acting as a ceRNA of miR-520c-3p in osteosarcoma cells. Cell Cycle. 2018;17 (13):1637-1648. doi:10.1080/15384101.2018.1489174

18. Lian Y, Li Z, Fan Y, et al. The IncRNA-HOXA-AS2/EZH2/LSD1 oncogene complex promotes cell proliferation in pancreatic cancer. Am J Transl Res. 2017;9(12):5496-5506.

19. Zhu X, Liu Y, Yu J, et al. LncRNA HOXA-AS2 represses endothelium inflammation by regulating the activity of NF-kappaB signaling. Atherosclerosis. 2019;281:38-46. doi:10.1016/j.atherosclerosis.2018. 12.012

20. Zong D, Li J, Cai S, et al. Notch1 regulates endothelial apoptosis via the ERK pathway in chronic obstructive pulmonary disease. Am J Physiol Cell Physiol. 2018;315(3):C330-C340. doi:10.1152/ ajpcell.00182.2017

21. Long YJ, Liu XP, Chen SS, Zong DD, Chen Y, Chen P. miR-34a is involved in CSE-induced apoptosis of human pulmonary microvascular endothelial cells by targeting Notch-1 receptor protein. Respir Res. 2018;19(1):21. doi:10.1186/s12931-018-0722-2

22. Global Initiative for Chronic Obstructive Lung Disease. Global strategy for diagnosis, management and prevention of chronic obstructive lung disease (updated 2013). Available from: http://goldcopd.org/. Accessed February 15, 2013.

23. De Smet EG, Mestdagh P, Vandesompele J, Brusselle GG, Bracke KR. Non-coding RNAs in the pathogenesis of COPD. Thorax. 2015;70(8):782-791. doi:10.1136/thoraxjnl-2014-206560

24. Soares Do Amaral N, Cruz EMN, de Melo Maia B, Malagoli Rocha R. Noncoding RNA profiles in tobacco- and alcohol-associated diseases. Genes (Basel). 2016;8(1):6. doi:10.3390/genes 8010006

25. Geisler S, Coller J. RNA in unexpected places: long non-coding RNA functions in diverse cellular contexts. Nat Rev Mol Cell Biol. 2013;14 (11):699-712. doi:10.1038/nrm3679

26. Uchida S, Dimmeler S. Long noncoding RNAs in cardiovascular diseases. Circ Res. 2015;116(4):737-750. doi:10.1161/CIRCRES AHA.116.302521

27. Feng SD, Yang JH, Yao $\mathrm{CH}$, et al. Potential regulatory mechanisms of IncRNA in diabetes and its complications. Biochem Cell Biol. 2017;95(3):361-367. doi:10.1139/bcb-2016-0110

28. Kopp F, Mendell JT. Functional classification and experimental dissection of long noncoding RNAs. Cell. 2018;172(3):393-407. doi:10.1016/j.cell.2018.01.011

29. Di Stefano A, Caramori G, Gnemmi I, et al. T helper type 17-related cytokine expression is increased in the bronchial mucosa of stable chronic obstructive pulmonary disease patients. Clin Exp Immunol. 2009;157(2):316-324. doi:10.1111/j.1365-2249.2009.03965.x

30. Huang P, Yan R, Zhang X, Wang L, Ke X, Qu Y. Activating Wnt/ beta-catenin signaling pathway for disease therapy: challenges and opportunities. Pharmacol Ther. 2019;196:79-90. doi:10.1016/j. pharmthera.2018.11.008 
31. Holtzman MJ, Byers DE, Alexander-Brett J, Wang X. The role of airway epithelial cells and innate immune cells in chronic respiratory disease. Nat Rev Immunol. 2014;14(10):686-698. doi:10.1038/ nri3739

32. Zhu Z, Gong W, Wang L, Bai Y, Yu Z, Zhang L. Efficient assessment of 3D train-track-bridge interaction combining multi-time-step method and moving track technique. Eng Struct. 2019;183:290-302. doi:10.1016/j.engstruct.2019.01.036

33. Chen Y, Luo H, Kang N, et al. Beraprost sodium attenuates cigarette smoke extract-induced apoptosis in vascular endothelial cells. Mol Biol Rep. 2012;39(12):10447-10457. doi:10.1007/s11033-0121924-1
34. Dabral S, Tian X, Kojonazarov B, et al. Notch1 signalling regulates endothelial proliferation and apoptosis in pulmonary arterial hypertension. Eur Respir J. 2016;48(4):1137-1149. doi:10.1183/ 13993003.00773-2015

35. Artavanis-Tsakonas S, Rand MD, Lake RJ. Notch signaling: cell fate control and signal integration in development. Science. 1999;284 (5415):770-776. doi:10.1126/science.284.5415.770

\section{Publish your work in this journal}

The International Journal of COPD is an international, peer-reviewed journal of therapeutics and pharmacology focusing on concise rapid reporting of clinical studies and reviews in COPD. Special focus is given to the pathophysiological processes underlying the disease, intervention programs, patient focused education, and self management protocols. This journal is indexed on PubMed Central, MedLine and CAS. The manuscript management system is completely online and includes a very quick and fair peer-review system, which is all easy to use. Visit http://www.dovepress.com/testimonials.php to read real quotes from published authors. 